\title{
Terahertz Imaging and Spectroscopy in Cancer Diagnostics: A Technical Review
}

\author{
Yan Peng, Chenjun Shi, Xu Wu, Yiming Zhu, and Songlin Zhuang \\ Terahertz Technology Innovation Research Institute, Shanghai Key Lab of Modern Optical System, Terahertz Science Cooperative \\ Innovation Center, University of Shanghai for Science and Technology, Shanghai Institute of Intelligent Science and Technology, \\ Tongji University, Shanghai, China
}

Correspondence should be addressed to Yiming Zhu; ymzhu@usst.edu.cn

Received 22 July 2020; Accepted 31 August 2020; Published 25 September 2020

Copyright @ 2020 Yan Peng et al. Exclusive Licensee Suzhou Institute of Biomedical Engineering and Technology, CAS. Distributed under a Creative Commons Attribution License (CC BY 4.0).

\begin{abstract}
Terahertz $(\mathrm{THz})$ waves are electromagnetic waves with frequency in the range from 0.1 to $10 \mathrm{THz}$. THz waves have great potential in the biomedical field, especially in cancer diagnosis, because they exhibit low ionization energy and can be used to discern most biomolecules based on their spectral fingerprints. In this paper, we review the recent progress in two applications of $\mathrm{THz}$ waves in cancer diagnosis: imaging and spectroscopy. $\mathrm{THz}$ imaging is expected to help researchers and doctors attain a direct intuitive understanding of a cancerous area. $\mathrm{THz}$ spectroscopy is an efficient tool for component analysis of tissue samples to identify cancer biomarkers. Additionally, the advantages and disadvantages of the developed technologies for cancer diagnosis are discussed. Furthermore, auxiliary techniques that have been used to enhance the spectral signal-to-noise ratio (SNR) are also reviewed.
\end{abstract}

\section{Introduction}

The early diagnosis of cancer is very important for patient treatment and recovery. If cancers can be confirmed and classified at an early stage, the patient survival rate can be greatly improved [1]. As shown in Figure 1, current clinical cancer diagnostic techniques can be divided into two catalogs: tissue imaging and spectroscopic biomarker detection.

Conventional imaging technologies, including magnetic resonance imaging (MRI), computed tomography (CT), and ultrasound (US), can be used to detect tissue structural differences, such as heterogeneity, cell apoptosis, and cell density, that are related to cancer [6]. Laboratory assays based on chromosomal analysis, methylation analysis, proteomic tools, immunological reactions, or antibody reactions [7] can be used to detect biomarkers (e.g., DNA or proteins) that are associated with cancer. However, to achieve high sensitivity and accuracy, these techniques mostly require large devices or complicated preprocessing steps.

Terahertz $(\mathrm{THz})$ waves are electromagnetic waves with frequency in the range from 0.1 to $10 \mathrm{THz}[8]$. As $\mathrm{THz}$ waves are located between the microwave and infrared regions on the electromagnetic spectrum, they exhibit features of both regions: they are nonionizing, noninvasive, and phasesensitive to polar substances, can be used for spectral fingerprinting, and coherent detection, and offer good resolution (up to $50 \mu \mathrm{m}$ ) and penetration capabilities [9]. Based on these unique features, $\mathrm{THz}$ waves have potential applications in many fields [10-15]. In oncology, the nonionizing and noninvasive nature and good penetration capabilities of $\mathrm{THz}$ waves make them suitable to inspect tissue both in vivo and ex vivo with high accuracy while causing minimal harm. Furthermore, spectral fingerprinting with $\mathrm{THz}$ waves can enable qualitative and quantitative analysis of cancer biomarkers.

$\mathrm{THz}$ spectroscopy is compared with other types of electromagnetic spectroscopy in terms of the information they can obtain in Table 1 [16-19]. THz spectroscopy detects the collective behavior (vibration and rotation) of molecules. Therefore, $\mathrm{THz}$ spectroscopy can be used to differentiate substances with different molecular structures as well as polymorph and chiral substances, even those that have the same elements and molecular bonds [20]. Although microwave spectroscopy can also detect molecular rotation in such substances, its wavelength is longer than that of $\mathrm{THz}$ wave spectroscopy, resulting in a relatively low resolution. 


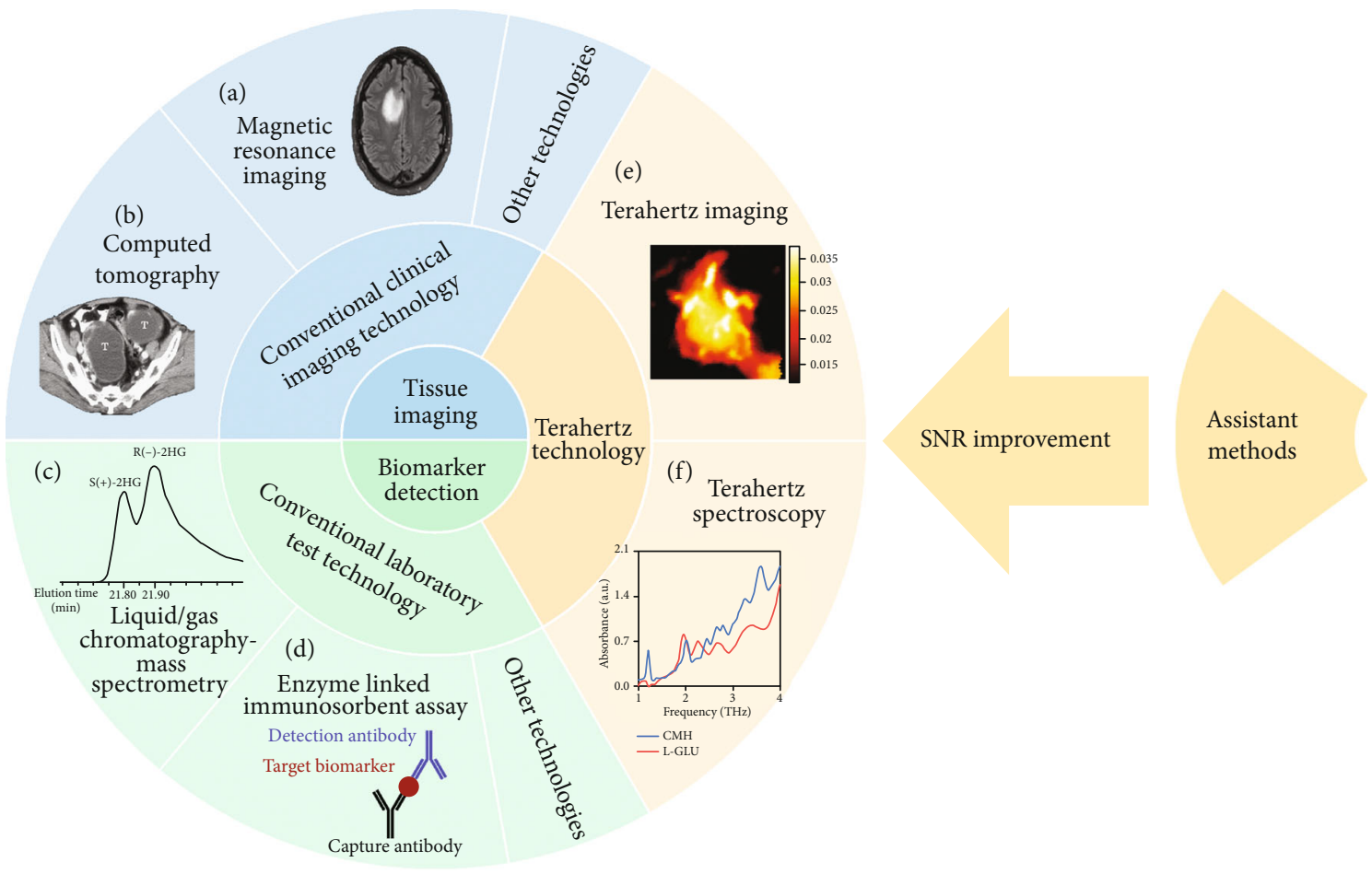

Conventional cancer diagnosis technology

(i) Advantage:

1) Specific analysis

2) High sensitivity

(ii) Disadvantage:

Systems or pre-treatment are too complicated and time consuming (unsuitbal for fast screening during surgery)
Terahertz technology

(i) Advantage:

1) Concise and compact system

2) Spectral fingerprinting

3) Fast screening

(ii) Disadvantage:

For actual usage, sensitivity needs further improvement

FIGURE 1: Multiple cancer diagnosis methods. (a) Magnetic resonance imaging of a brain tumor (from Ref. [2]). (b) Computed tomography scan of bilateral serous cystadenocarcinomas in a 50-year-old woman with contrast enhancement shows bilateral ovoid tumors (T) with septa and mural nodules (from Ref. [3]). (c) Basic principle of the enzyme-linked immunosorbent assay: the capture antibody captures the target biomarker then binds to the detection antibody. (d) Separation of the (R) and (S) enantiomers of the cancer biomarker $2 \mathrm{HG}$ by liquid chromatography-mass spectrometry (from Ref. [4]). (e) Terahertz imaging of a breast tumor (from Ref. [5]). (f) Terahertz absorption spectra of creatine (red line) and L-Glutamic acid (blue line).

TABLE 1: Information detected by different electromagnetic spectroscopies.

\begin{tabular}{lc}
\hline Spectroscopy type & Detectable information \\
\hline $\begin{array}{l}\text { Energy-dispersive } \\
\text { X-ray spectroscopy }\end{array}$ & Elemental composition \\
Microwave spectroscopy & Rotation of molecules \\
Raman/infrared spectroscopy & $\begin{array}{c}\text { Fundamental vibration } \\
\text { of chemical bonds }\end{array}$ \\
Ultraviolet-visible light & $\begin{array}{c}\text { Electron excitation of } \\
\text { specific molecules }\end{array}$ \\
Terahertz spectroscopy & $\begin{array}{c}\text { Collective behavior of molecules } \\
\text { (vibration and rotation) }\end{array}$ \\
\hline
\end{tabular}

Given the above advantages of $\mathrm{THz}$ technologies and considering that they are generally compact systems, $\mathrm{THz}$ technologies have the potential to be applied for rapid tissue imaging during surgery. For cancer tissue imaging, THz imaging can distinguish cancerous and peritumoral tissue from normal tissue with clear boundaries, thus providing more intuitive information to the surgeon to aid in the removal of the cancerous and peritumoral tissue. However, the penetration depth of $\mathrm{THz}$ waves in fresh tissue is limited to the water absorption depth (about $276 \mu \mathrm{m}$ for cancer tissue at $0.5 \mathrm{THz}$ ) $[21,22]$. Therefore, current studies of $\mathrm{THz}$ imaging for cancer mainly focus on imaging excised tissue or using reflection imaging systems to study the surface layer of tissue, both of which can reduced the influence of water absorption. Furthermore, current studies of $\mathrm{THz}$ imaging are based mainly on differences in water content, which can only be used as a reference instead of a specific identification [Reference].

Biomarker detection can be helpful in the diagnosis of cancer. As different substances have different THz spectra (i.e., spectral "fingerprints"), THz spectroscopy can be applied to realize fast and accurate identification of biomarkers in cancer 
TABLE 2: Current ex vivo $\mathrm{THz}$ imaging studies.

\begin{tabular}{|c|c|c|c|}
\hline Authors & THz system & Imaging target & Results \\
\hline $\begin{array}{l}\text { Martin et al., } \\
2016[24]\end{array}$ & $\begin{array}{l}\text { A continuous-wave } \mathrm{THz} \\
\text { imaging system working } \\
\text { at } 0.584 \mathrm{THz} \text { with } \\
\text { circular polarization }\end{array}$ & $\begin{array}{l}\text { Fresh tumor and normal human } \\
\text { skin tissue specimens }\end{array}$ & $\begin{array}{l}\text { Contrast between cancerous and } \\
\text { normal tissues was found with a } \\
\text { resolution of } 0.15 \mathrm{~mm}\end{array}$ \\
\hline $\begin{array}{l}\text { Bowman et al., } \\
2016[25]\end{array}$ & $\begin{array}{l}\text { A pulsed } \mathrm{THz} \text { imaging and } \\
\text { spectroscopy system }\end{array}$ & $\begin{array}{c}\text { Excised paraffin-embedded breast } \\
\text { tissue with breast invasive } \\
\text { ductal carcinoma }\end{array}$ & $\begin{array}{c}\text { The carcinoma areas exhibited lower } \\
\text { transmission and higher reflection } \\
\text { than normal areas as defined } \\
\text { based on pathology }\end{array}$ \\
\hline $\begin{array}{l}\text { Yamaguchi et al., } \\
2016 \text { [26] }\end{array}$ & $\begin{array}{l}\text { A reflection } \mathrm{THz} \text { time-domain } \\
\text { spectroscopy system }\end{array}$ & $\begin{array}{l}\text { Fresh and paraffin-embedded } \\
\text { tissues from a rat glioma model }\end{array}$ & $\begin{array}{l}\text { A difference of } 0.02(0.8-1.5 \mathrm{THz}) \\
\text { in the refractive index was found } \\
\text { between glioma and normal area }\end{array}$ \\
\hline $\begin{array}{l}\text { Wahaia et al., } \\
2016 \text { [27] }\end{array}$ & $\begin{array}{l}\text { A continuous-wave } \mathrm{THz} \\
\text { imaging system working } \\
\text { at } 0.59 \mathrm{THz}\end{array}$ & Dehydrated human colon tissues & The imaging resolution reached $500 \mu \mathrm{m}$ \\
\hline $\begin{array}{l}\text { Grootendorst et al., } \\
2017 \text { [28] }\end{array}$ & $\begin{array}{l}\text { A handheld } \mathrm{THz} \text { pulsed } \\
\text { imaging system }\end{array}$ & $\begin{array}{l}\text { Freshly excised breast } \\
\text { cancer samples }\end{array}$ & $\begin{array}{c}\text { The identification accuracy of cancerous } \\
\text { areas reached } 75 \%\end{array}$ \\
\hline $\begin{array}{l}\text { Bowman et al., } \\
2018 \text { [29] }\end{array}$ & $\begin{array}{l}\text { A pulsed THz imaging and } \\
\text { spectroscopy system }\end{array}$ & $\begin{array}{l}\text { Freshly excised murine xenograft } \\
\text { breast cancer tumors }\end{array}$ & $\begin{array}{l}\text { Comparison with pathology results } \\
\text { showed an accuracy above } 80 \%\end{array}$ \\
\hline $\begin{array}{l}\text { Cassar et al., } \\
2018[30]\end{array}$ & $\begin{array}{l}\text { A pulsed } \mathrm{THz} \text { imaging system } \\
\text { with a reflection mode }\end{array}$ & Freshly excised breast tissue & The spatial resolution reached $1 \mathrm{~mm}$ \\
\hline $\begin{array}{l}\text { Vohra et al., } \\
2018[31]\end{array}$ & $\begin{array}{l}\text { A pulsed } \mathrm{THz} \text { imaging system } \\
\text { with a reflection mode }\end{array}$ & $\begin{array}{l}\text { Freshly excised and formalin/ } \\
\text { paraffin-fixed breast tumor } \\
\text { tissues from a mouse model }\end{array}$ & $\begin{array}{l}\text { Cancerous areas exhibited the highest } \\
\text { reflection and agreed with the } \\
\text { pathology results }\end{array}$ \\
\hline $\begin{array}{l}\text { Yeo et al., } \\
2019 \text { [32] }\end{array}$ & $\begin{array}{l}\text { A pulsed } \mathrm{THz} \text { imaging system } \\
\text { with a reflection mode }\end{array}$ & $\begin{array}{l}\text { Paraffin-embedded malignant } \\
\text { tissues in human lung and } \\
\text { small intestine tissues }\end{array}$ & $\begin{array}{l}\text { The adipose tissue area showed a } \\
\text { lower refractive index and with a } \\
\text { diffraction-limited spot size } \\
\text { of } \sim 360 \mu \mathrm{m} \text { at } 1 \mathrm{THz}\end{array}$ \\
\hline $\begin{array}{l}\text { Okada et al., } \\
2019[33]\end{array}$ & $\begin{array}{l}\text { A scanning laser } \mathrm{THz} \text { near-field } \\
\text { reflection imaging system }\end{array}$ & $\begin{array}{l}\text { Paraffin-embedded human } \\
\text { breast tissue }\end{array}$ & The spatial resolution reached $20 \mu \mathrm{m}$ \\
\hline $\begin{array}{l}\text { Bowman et al., } \\
2019[34]\end{array}$ & $\begin{array}{l}\text { A pulsed } \mathrm{THz} \text { imaging and } \\
\text { spectroscopy system }\end{array}$ & $\begin{array}{l}\text { Freshly excised breast } \\
\text { cancer tumors }\end{array}$ & $\begin{array}{l}\text { The cancerous areas exhibited higher } \\
\text { absorption coefficients and refractive } \\
\text { indexes than normal tissues, and } \\
\text { the resolution reached } 200 \mu \mathrm{m}\end{array}$ \\
\hline
\end{tabular}

tissue. Furthermore, THz spectroscopy can be combined with various algorithms to realize the quantitative analysis of cancer biomarkers, which may be a potential tool for rapid cancer staging. However, in most cases, various substances (such as water, proteins, fat, fiber, and other organic components) are also present in the tissue, and the biomarker concentration is usually very low. These conditions result in a low spectral SNR, which gives rise to absorption peaks that cannot be readily identified [23]. Therefore, current THz-spectroscopy-based studies of biomarkers are mostly done on pure biomarkers. If we can construct $\mathrm{THz}$ images based on the resonance peaks of biomarkers, cancerous areas can be identified more accurately. However, further development is required to realize this objective as current imaging systems with high-power continuouswave $\mathrm{THz}$ sources can only generate $\mathrm{THz}$ waves at a fixed frequency, and pulsed $\mathrm{THz}$ sources cannot provide enough spectral power. Thus, both $\mathrm{THz}$ imaging and spectroscopy are limited by source energy, leading to poor SNR in the spectral results. To address this issue, some researchers are working to enhance the spectral SNR, which is expected to help detect biomarkers in mixed samples.
In this paper, we summarize recent studies of $\mathrm{THz}$ imaging and spectroscopy in cancer diagnosis from the past 5 years. We also present auxiliary methods to improve the SNR or enhance the $\mathrm{THz}$ signal. These works demonstrate the promise of using $\mathrm{THz}$ technologies for cancer diagnosis.

\section{Terahertz Imaging in Cancer Diagnosis}

Given the molecular fingerprinting and nonionizing features of $\mathrm{THz}$ waves, $\mathrm{THz}$ imaging can realize accurate and safe tissue imaging. However, because $\mathrm{THz}$ waves are readily absorbed by water, cancer tissue is generally studied ex vivo (e.g., as freshly excised tissue or paraffin-embedded tissue) after cutting into slices that $\mathrm{THz}$ waves can penetrate. Table 2 shows the ex vivo $\mathrm{THz}$ imaging studies that have been conducted during the past 5 years. The resolution of $\mathrm{THz}$ imaging is improving with recent research advancements, particularly the development of near-field imaging methods.

Figure 2 shows frequency-domain images of an infiltrating ductal carcinoma at $1 \mathrm{THz}$ from Bowman et al. (2016), where cancerous areas exhibited lower transmission and 


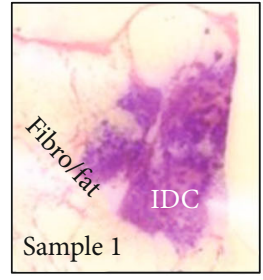

(a)

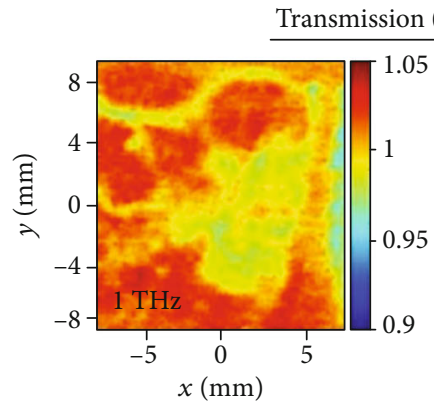

(c)

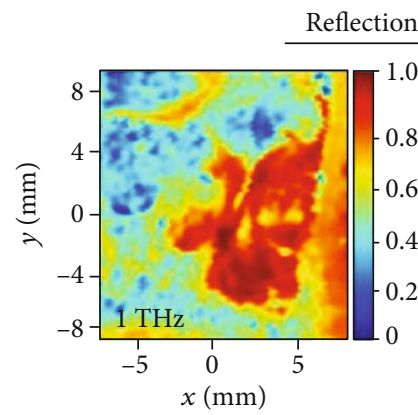

(e)

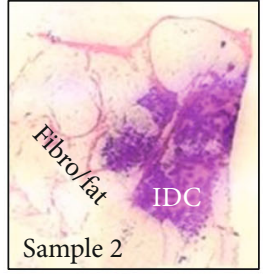

(b) ig. 3)

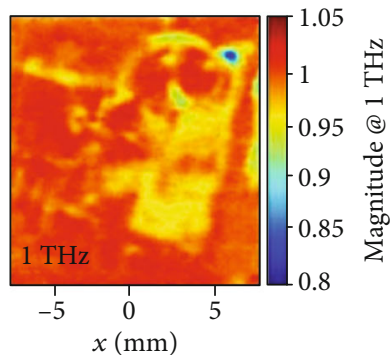

(d)
Figure 2: Frequency-domain images of infiltrating ductal carcinoma. Pathology for (a) Sample 1 and (b) Sample 2. Transmission magnitude images at $1 \mathrm{THz}$ of (c) Sample 1 and (d) Sample 2. Reflection magnitude images at $1 \mathrm{THz}$ of (e) Sample 1 and (f) Sample 2 (from Ref. [25]).

higher reflection compared with the adjacent normal areas of fibrous and fatty tissues [25]. Similar observations were made in many other studies. The observed contrast was attributed to higher water content in the cancerous areas, which resulted in higher absorption, reflection, or refractive index with $\mathrm{THz}$ waves.

For application in rapid cancer screening during surgery, imaging systems must be compact and easy to use. The handheld $\mathrm{THz}$ pulsed imaging (TPI) system designed by Grootendorst et al. (2017) shown in the schematic illustration in Figure 3 is a good example of such a system [28]. In this TPI system, the quartz tip scans an area of $15 \times 2$ $\mathrm{mm}$, and measurements are collected in 26 pixels. Using this system, the identification accuracy for cancerous areas reached $75 \%$. However, one limitation of this ex vivo study is that the sample needs to be sliced into sections of uniform thickness (often on the order of micrometers) to ensure that $\mathrm{THz}$ waves can penetrate them.

As the human body contains various components, including large amounts of water, $\mathrm{THz}$ waves cannot pene- trate deeply. Therefore, most studies rely on ex vivo tissue imaging and few have used in vivo tissue imaging.

In the past 5 years, only two studies have successfully obtained in vivo images by using a pulsed $\mathrm{THz}$ reflectometric imaging system. In 2016, Ji et al. obtained images of gliomas from live tumor model mice with a resolution of $250 \mu \mathrm{m}$. In these images, the tumor tissue exhibited a higher intensity signal than the adjacent normal tissue [35]. In 2019, Wu et al. also obtained images of brain gliomas in model mice with a resolution of $200 \mu \mathrm{m}$ by using a $\mathrm{THz}$ reflection imaging system with $2.52 \mathrm{THz}$ continuous waves [36]. Currently, in vivo $\mathrm{THz}$ imaging research is still in the beginning stage. For studies in the human body, two critical issues remain. First, human tissue contains significantly more types of components compared with mouse tissue, which complicated spectral analysis. Second, individual differences in tissue contents will result in differences in absorption and refraction of $\mathrm{THz}$ waves between subjects. These two issues seriously interfere with the accurate identification of cancerous areas.

Currently, $\mathrm{THz}$ images of cancer tissue are mostly constructed based on differences between the water contents of normal tissue and cancer tissue. Although this can elucidate differences between the cancer tissue and the adjacent area, which gives an intuitive understanding of the cancer tissue, it cannot be used to diagnose the specific cancer type.

\section{Terahertz Spectroscopy in Cancer Diagnosis}

Most biomolecules exhibit unique resonance (i.e., spectral "fingerprints") in the presence of $\mathrm{THz}$ waves. Thus, THz spectroscopy can be used to identify many cancer biomarkers and, therefore, determine the type of cancer. However, in most cases, the biomarker concentration in cancer tissues is small, and the tissue contains hundreds of other substances [37], which makes it difficult to identify the absorption peaks of a biomarker in mixed spectra. Therefore, most current studies examine the biomarkers in their pure state or in simple mixtures (e.g., with ten different substances). To realize cancer detection basing on the spectral fingerprints of biomarkers, spectroscopy systems need to be further developed to achieve higher signal intensity and sensitivity.

3.1. Identification of Biomarkers in Cancer. To study the biomarkers in cancer tissue, the first step should be to study the biomarkers themselves. Many research groups have identified the characteristic peaks of biomarkers for different diseases. However, the complicated molecular structures of cancer-related DNA and proteins cannot yet be identified using $\mathrm{THz}$ spectroscopy. Smaller molecules are easier to identify and, therefore, have low identification errors; thus, they are the preferred targets for study. $\boldsymbol{\gamma}$-Aminobutyric acid exists in the central nervous system in various animal species, and its concentration markedly decreases in cancer tissue [38]. Cheng et al. examined the spectrum of $\gamma$-aminobutyric acid from 0.5 to $18 \mathrm{THz}$ and found absorption peaks at 1.13 , $1.52,2.03,2.58,3.48,4.34,5.53,7.80,8.26,9.63,12.0$, and $16.7 \mathrm{THz}$ [39]. The neuronal loss caused by tumors also leads to a decrease in $\mathrm{N}$-acetyl-aspartate [40], which exhibits absorption peaks at 1.466, 1.695, 1.979, and $2.879 \mathrm{THz}$. Chen 


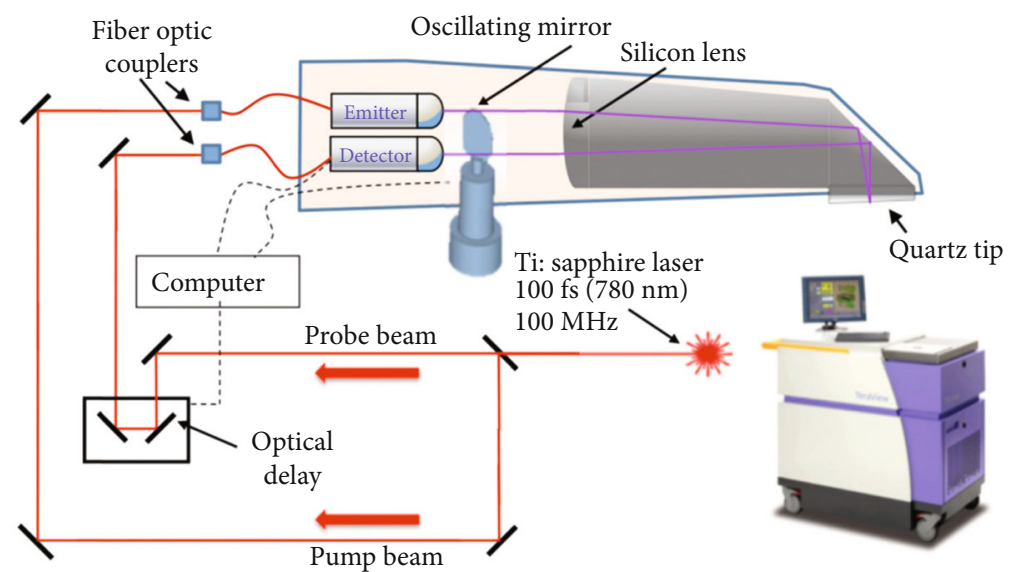

Figure 3: Schematic illustration of the handheld TPI probe system (from Ref. [28]).

et al. also reported the $\mathrm{THz}$ spectra of the glioma biomarker 2-hydroxyglutaric acid disodium salt ( $2 \mathrm{HG})$, which exists as two different isomers with different spectra: L-2HG exhibits characteristic peaks at $0.769,1.337,1.456$, and $1.933 \mathrm{THz}$, while D-2HG exhibits characteristic peaks at $0.760,1.200$, 1.695, and 2.217 THz. Therefore, $\mathrm{THz}$ spectroscopy can be used to accurately and rapidly identify both isomers of $2 \mathrm{HG}$, so it may be used as a tool for the early diagnosis of glioma [41]. Yang et al. measured the $\mathrm{THz}$ spectrum of myo-inositol, which is found in elevated levels in gliomatosis [42], and reported that it exhibits four characteristic peaks at $1.00,1.46,1.58,1.85$, and $2.05 \mathrm{THz}$ [43].

These findings showing the resonance absorption peaks of several cancer-related biomolecules in the $\mathrm{THz}$ range demonstrate that $\mathrm{THz}$ spectroscopy can be a potential tool for biomarker detection in cancer diagnosis. However, because the biomarkers are found in low concentrations and obscured by hundreds of other substances that are also present in the tissue, the accurate $\mathrm{THz}$-based detection of biomarkers is extremely difficult using existing technologies.

3.2. $\mathrm{THz}$ Spectral Algorithms for Cancer Diagnosis. Because the changes in biomarker concentration intensify with the development of cancer, the quantitative analysis of biomarker concentrations can help with cancer staging [44]. Various regression algorithms have been used to realize quantitative biomarker analysis. However, as mentioned before, it is currently difficult to identify biomarkers in tissue samples. Therefore, most of these preliminary component analysis studies used engineered samples with no more than ten substances.

In 2016, Ge et al. constructed linear and nonlinear models to quantitatively analyze the concentration of aflatoxin B1, which is a carcinogen. They combined $\mathrm{THz}$ spectroscopy with the partial least squares (PLS), principal component regression (PCR), support vector machine (SVM), and PCA-SVM methods. The results showed that the PLS and PCR models provided the best aflatoxin B1 quantification results in the concentration range of $1-$ $50 \mu \mathrm{g} / \mathrm{mL}$; the accuracy was as high as $87.5 \%$. In the concentration range of $1-50 \mathrm{ng} / \mathrm{mL}, \mathrm{SVM}$ and PCA-SVM showed the best results, and the accuracy reached 93.75\% [45]. Lu et al. qualitatively and quantitatively analyzed binary amino acid mixtures using PLS and interval partial least square (iPLS) regressions. The iPLS regression resulted in a lower root mean square error $(0.39 \%)$ for the two components than the PLS method [46]. In 2018, Peng et al. reported a method to analyze the main substances in human brain tissue cells, including $\gamma$-aminobutyric acid, L-glutamic acid, D-myo-inositol, creatine monohydrate, cholesterol, noradrenaline, and $\mathrm{N}$-acetylaspartate. When cancer occurs, the concentrations of noradrenaline and $\mathrm{N}$-acetylaspartate will increase and decrease, respectively. A diagram of the proposed algorithm is shown in Figure 4. The algorithm comprises three steps: wavelet transform for denoising, polynomial fitting for to remove the baseline signal, and support vector regression (SVR) modeling for analysis. This approach was shown to predict the concentration of these two substances among all seven substances with a root mean square error of $0.40 \%$ [47].

Algorithms have been also used to differentiate cancerous tissue from normal tissue based on their spectral differences. A few studies have succeeded in identifying cancer in real tissue samples rather than engineered mixtures. In 2015, Qi et al. combined $\mathrm{THz}$ spectroscopy with a fuzzy rulebuilding expert system (FuRES) and fuzzy optimal associative memory (FOAM) for the diagnosis of cervical carcinoma. In a test on 52 cervical tissue sections ( 32 normal and 20 cancerous), the classification accuracy of the FuRES reached $92.9 \% \pm 0.4 \%$, and that of FOAM reached $92.5 \% \pm 0.4 \%$ [48]. In 2018, Liu et al. combined $\mathrm{THz}$ spectroscopy with two linear compression methods (principal component analysis and locality preserving projections (LPP)) and a nonlinear method (Isomap) for the identification of hepatic tumors. The best classification accuracies from twodimensional time-domain data were achieved with an Isomap probabilistic neural network (PNN; 99.81\% $\pm 0.30 \%$ ) and an Isomap support vector machine (SVM; $99.69 \% \pm$ $0.61 \%)$. The best classification results from twodimensional frequency-domain data were achieved by LPPPNN (100.00\% $\pm 0.00 \%)$ and LPP-SVM $(99.75 \% \pm 0.32 \%)$ [49]. In 2020, Liu et al. proposed a method of wavelet entropy feature extraction and a machine learning classifier 


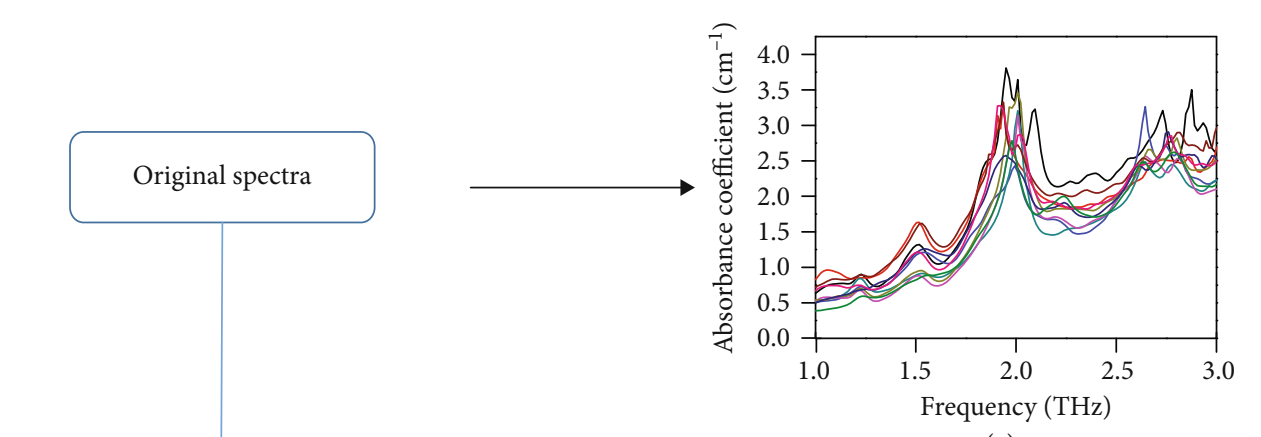

(a)

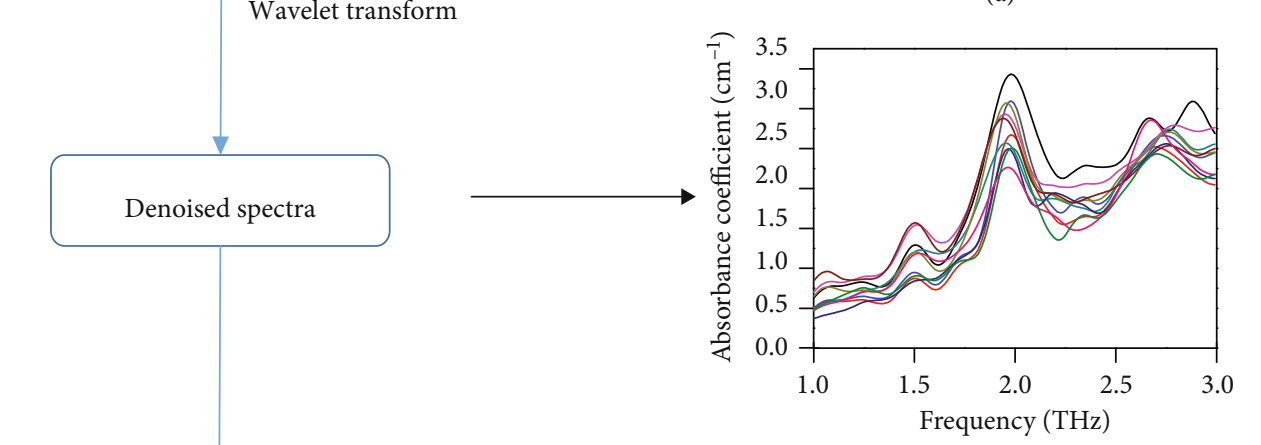

(b)
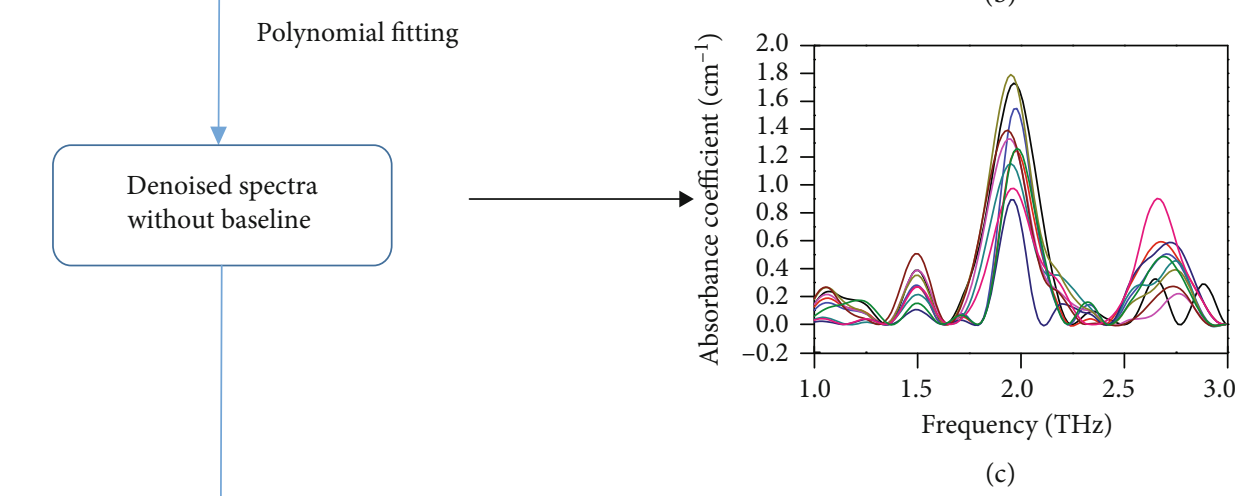

(c)

SVR modeling

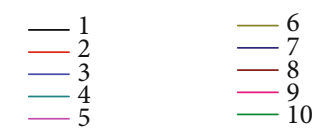

Qualitative and quantitative analysis

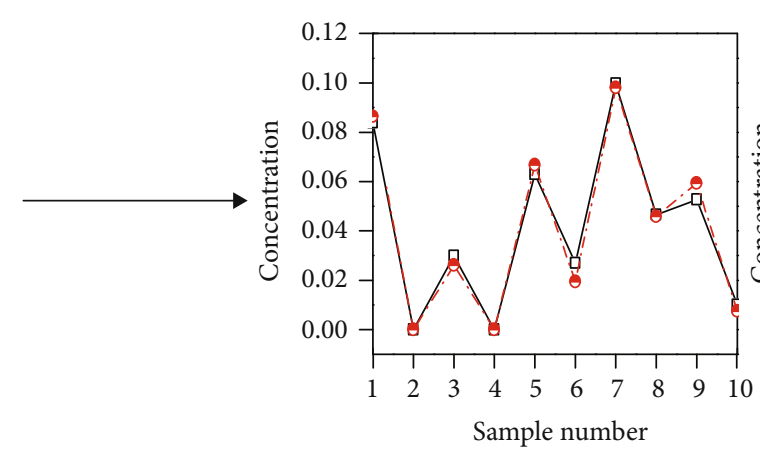

(d)

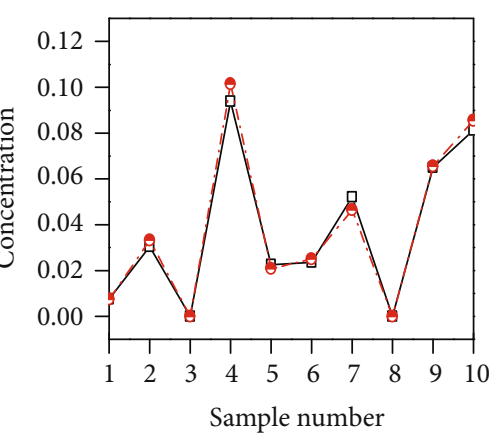

(e)

$\rightarrow-$ Predicted concentration

- $\bullet$ Real concentration

Figure 4: Diagram of the proposed analysis algorithm. (a) The original spectra of ten samples with seven components in different proportions. (b) The denoised spectra of samples processed by wavelet transform. (c) The denoised spectra without baseline after processing by polynomial fitting. (d) Actual and predicted concentrations of N-acetylaspartate. (e) Actual and predicted concentrations of noradrenaline (from Ref. [47]). 
for the recognition of breast invasive ductal carcinoma from $\mathrm{THz}$ pulsed signals. Results showed that cancer identification was achieved with a precision of $92.85 \%$ [50].

These studies demonstrated the feasibility of using various algorithms in combination with $\mathrm{THz}$ spectroscopy to diagnose cancer. However, the key remaining issue is that the identification accuracy will decrease with increasing numbers of components in the mixture. Therefore, the algorithms for qualitative analysis of biomarkers are still not applicable for tissue samples. To solve this problem, some groups proposed different methods.

\section{Methods of SNR Enhancement in THz Measurements for Cancer Diagnosis}

As mentioned above, one of the critical issues associated with $\mathrm{THz}$ detection of biomarkers for cancer diagnosis is that tissue strongly absorbs $\mathrm{THz}$ waves, which leads to a poor SNR in the obtained spectra. Therefore, several research groups have used different methods to enhance the SNR of THz spectra.

Some groups have used contrast agents to enhance the contrast of cancerous areas. In 2016, Zhang et al. proposed the use of superparamagnetic iron oxide nanoparticles (SPIOs) to increase the reflection of $\mathrm{THz}$ waves in water upon exposure to an alternating magnetic field. Focal-plane imaging experiments were conducted on water with and without $4 \mathrm{~g} / \mathrm{L}$ SPIOs, and the average amplitude of the relative reflection was $29.41 \% \pm 0.42 \%$ in water with SPIOs compared with only $0.30 \% \pm 0.03 \%$ in water without SPIOs. As the imaging contrast between cancerous and normal tissues depends on the difference in the water contents in these tissues, the SPIOs can enhance the contrast of cancerous areas relative to normal areas [51]. In 2017, Bowman et al. proposed the use of nanometer-scale onion-like carbon (OLC) that can be activated for selective binding to cancer cells to increase the reflection of cancerous tissues. Experimental results showed that an invasive ductal carcinoma phantom with $10 \%$ OLC exhibited drastically higher reflection compared with the surrounding fibroglandular (healthy) tissues [52]. In 2019, Huang et al. designed silica-coated gold nanorods as a contrast agent for imaging analyzing prostate cancer cells. The intensity of the cancerous area with silica-coated gold nanorods was $25.35 \%$ higher than that of the sample without nanoparticles [53].

Optical clearing agents have been used to reduce the water inside the issue for $\mathrm{THz}$ spectroscopy, reducing the absorption of $\mathrm{THz}$ waves to improve the spectral SNR and allow for easier identification of biomarkers. In 2018, Musina et al. proposed several $\mathrm{THz}$ wave penetration-enhancing agents for optical clearing of tissues: polyethylene glycol with different molecular weights, propylene glycol, ethylene gly$\mathrm{col}$, and dimethyl sulfoxide. These agents have about three times lower absorption than water [54]. Yang et al. used fluorinated oil as an optical clearing agent to replace the liquid medium around living cells. An independent $t$-test revealed that the difference between the absorption of fluorinated oil with and without cells was statistically significant $(P<0.05$ at $0.5,1.0$, and $1.5 \mathrm{THz}$ ) [55]. These studies demonstrated that optical clearing agents can be used to reduce the noise from water absorption, which enhances the spectral SNR and allows biomarkers to be identified more effectively.

Antibody-based biosensors can be used for the detection of cancer cells. Figure 5 shows a biosensor designed by Hassan et al. that comprises a silicon dioxide layer coated with aptamers [56]. The biosensor was used to detect metastatic breast cancer cells as follows: the antibody binds only to cancer-related cell surface protein, changing the $\mathrm{THz}$ absorption of these cancer cells relative to normal cells. The experimental results showed that the $\mathrm{THz}$ amplitude changed linear change with the cancer stage. The detection limit was one breast cancer cell in a $100 \mu \mathrm{L}$ volume. This approach is a good method for detecting biomarkers that do not have characteristic absorption peaks in the $\mathrm{THz}$ band. However, the amplitude of a $\mathrm{THz}$ signal may be influenced by the environment (such as humidity), which may cause jitter in the signal and lead to error.

To minimize the error and further enhance the spectral SNR, metamaterials have been designed as biosensors. These metamaterials exhibit resonance peaks with high spectral SNR in the THz range. The peaks exhibit frequency shifts according to the refractive index of the sample that the metamaterial is in contact with. Thus, these metamaterials can be used in combination with antibodies to detect cancer biomarkers or cells. In 2016, Geng et al. demonstrated metamaterials with specific antibodies to detect two biomarkers of liver cancer: alphafetoprotein and glutamine transferase isozyme II. This biosensor exhibited detection limits of $5 \mathrm{mu} / \mathrm{mL}$ and $0.02524 \mu \mathrm{g} / \mathrm{mL}$ for alphafetoprotein and glutamine transferase isozyme II, respectively [57]. In 2018, Liu et al. developed a bow-tie THz metamaterial biosensor coated with an antibody for the epidermal growth factor receptor (EGFR), which is a biomarker for gastrointestinal cancer, breast cancer, head-and-neck cancer, and epithelial cancer. This biosensor exhibited a detection limit of $10 \mathrm{fmol} / \mathrm{mL}$ [58]. In 2020, Weisenstein et al. designed a metamaterial biosensor combined with complementary DNA strands that bind specifically to single-stranded oligo- or poly-nucleotide probe molecules. This biosensor was used for the detection of a human tumor marker, melanoma inhibitory activity (MIA) mRNA, with a detection limit of $1.55 \times 10^{-12} \mathrm{~mol} / \mathrm{L}$ [59].

These proposed auxiliary methods all succeeded in enhancing the spectral SNR. Contrast agents, optical cleaning agents, antibodies, and metamaterials can specifically elevate or eliminate the signals of certain substances, thus enhancing the final spectral SNR. However, sample contamination with additional substances is unavoidable. Furthermore, substances for in vivo use must not cause harm to the human body. Antibodies and metamaterials can be used in combination to enhance the detection sensitivity and identify biomarkers in low concentrations. However, the process of searching for antibodies is not easy, and there are no antibodies available for some cancer biomarkers.

\section{Conclusion}

In this article, we reviewed many studies conducted in the past 5 years that focus on $\mathrm{THz}$ imaging and $\mathrm{THz}$ spectroscopy applied in cancer diagnosis. THz imaging can clearly 


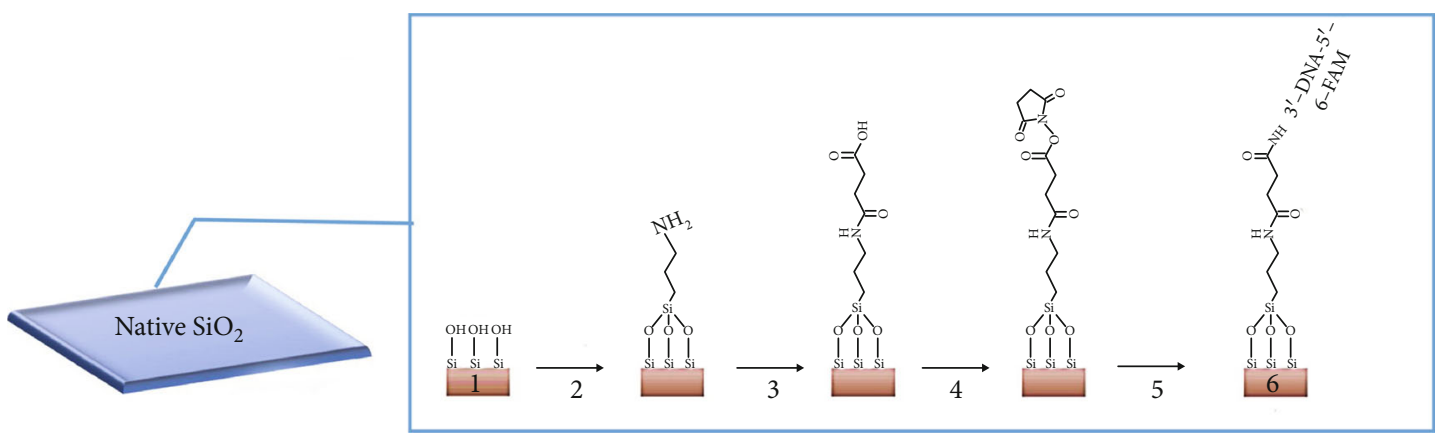

(a)

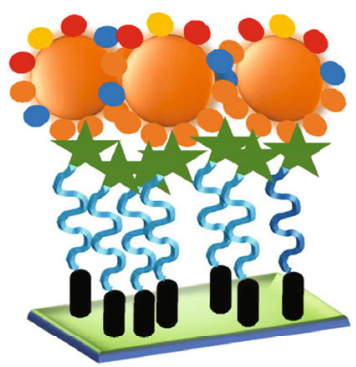

Cancer cells (MCF7) on MAMB1 sensing plate

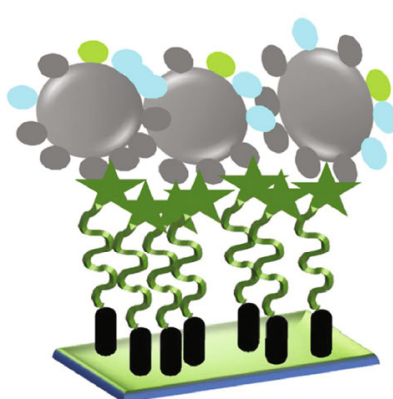

Cancer cells (MDA-MB-415) MAMA2 sensing plate

The native $\mathrm{SiO}_{2}$ layer on the sensing plate before (blue) modification and after (green)

$\mathrm{NH}_{2}$ on the 3 prime end of each aptamer

6-FAM fluorescence

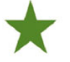

labelled on the 5 prime of each aptamer

MAMB1 (blue) and MAMA2 (green) aptamers

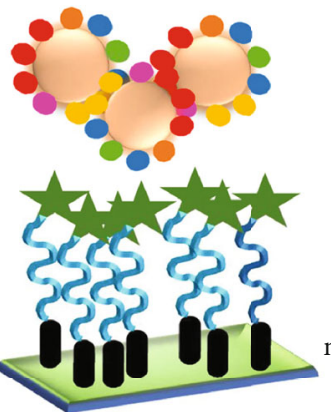

Normal cells (MCF10A) on MAMB1 sensing plate

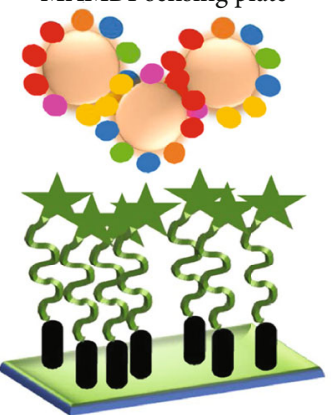

Normal cells (MCF10A) on MAMA2 sensing plate

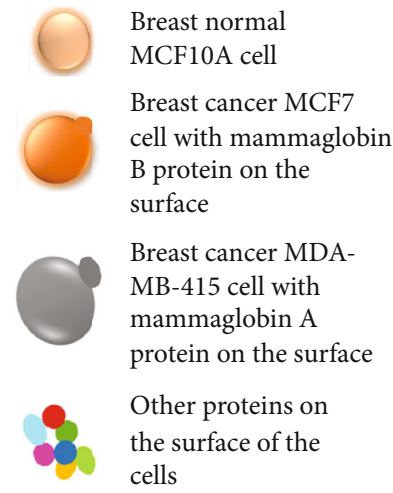

(b)

Figure 5: Schematic diagram of the experimental design of an antibody-based biosensor. (a) Modification of the $\mathrm{SiO}_{2}$ layer to immobilize MAMB1 and MAMA2 aptamers. (b) After immobilization, different numbers of cells were added to each sensing plate, and the THz amplitude was recorded (from Ref. [56]).

differentiate cancerous tissue from normal tissue with clear boundaries based on the difference in the water contents of these tissues. However, because of the high absorption of
THz waves by water and other substances present in tissue, the penetration depth of $\mathrm{THz}$ waves is limited to $1 \mathrm{~mm}$. Therefore, current THz imaging studies in oncology focus 
on excised tissue or use reflection imaging systems to study the surface layer of the tissue. Furthermore, $\mathrm{THz}$ imaging based only on water content cannot be used to determine the type of cancer.

Biomarkers can be used for cancer identification in most cases. Some biomarkers exhibit resonance with $\mathrm{THz}$ waves, so $\mathrm{THz}$ spectroscopy can be a tool for biomarker identification in cancer tissue. However, the low concentrations of biomarkers and the presence of various outer substances in the cancer tissue make it difficult to identify biomarkers in real tissues. Therefore, current $\mathrm{THz}$ spectroscopy technologies can only identify biomarkers in engineered mixtures with no more than ten substances.

Auxiliary methods have been developed to address these issues. These methods included the use of contrast agents, optical cleaning agents, antibodies, and metamaterials to enhance the spectral SNR. However, sample contamination by additional substances is unavoidable. Antibodies have been combined with metamaterial biosensors to achieve highly sensitive biomarker detection. However, the process of developing antibodies for biomarkers is complicated, and antibodies may not be available for some cancer biomarkers.

In summary, the studies presented here have demonstrated the feasibility of using $\mathrm{THz}$ technologies in cancer diagnosis. However, additional development is needed before these technologies can be practically applied for the early diagnosis of cancer. Future developments of THz technologies for cancer diagnosis include combining $\mathrm{THz}$ imaging with $\mathrm{THz}$ spectral fingerprinting of biomarkers to realize qualitative identification and quantitative analysis simultaneously. To achieve this objective, further improvements in $\mathrm{THz}$ systems and auxiliary methods are needed.

\section{Data Availability}

The datasets used and/or analyzed during the current study are available from the corresponding author on reasonable request.

\section{Conflicts of Interest}

The authors have declared no conflicts of interest.

\section{Authors' Contributions}

Y. P. and Y.M. Z. developed the idea and supervised the project. C.J. S. contributed to the research of current studies. Y. P. and C.J. S. were major contributors in writing the manuscript. All authors revised the paper and approved the final manuscript.

\section{Acknowledgments}

This study was supported by the National Major Project of Scientific Instrument and Equipment Development (2017YFF0106300), the National Natural Science Foundation of China (61922059, 61771314, and 81961138014), the 111 Project (D18014), the International Joint Lab Program supported by Science and Technology Commission Shanghai
Municipality (17590750300), and the Key project supported by Science and Technology Commission Shanghai Municipality (YDZX20193100004960).

\section{References}

[1] E. Robinson, J. Mohilever, J. Zidan, and D. Sapir, "Delay in diagnosis of cancer. Possible effects on the stage of disease and survival," Cancer, vol. 54, no. 7, pp. 1454-1460, 1984.

[2] R. P. Joseph, C. S. Singh, and M. Manikandan, "Brain tumor MRI image segmentation and detection in image processing," International Journal of Research in Engineering and Technology, vol. 3, no. 1, pp. 1-5, 2014.

[3] S. E. Jung, J. M. Lee, S. E. Rha, J. Y. Byun, J. I. Jung, and S. T. Hahn, "CT and MR imaging of ovarian tumors with emphasis on differential diagnosis," Radiographics, vol. 22, no. 6, pp. 1305-1325, 2002.

[4] P. S. Ward, J. Patel, D. R. Wise et al., "The common feature of leukemia-associated IDH1 and IDH2 mutations is a neomorphic enzyme activity converting $\alpha$-ketoglutarate to 2 hydroxyglutarate," Cancer Cell, vol. 17, no. 3, pp. 225-234, 2010.

[5] A. J. Fitzgerald, V. P. Wallace, M. Jimenez-Linan et al., “Terahertz pulsed imaging of human breast tumors," Radiology, vol. 239, no. 2, pp. 533-540, 2006.

[6] R. García-Figueiras, S. Baleato-González, A. R. Padhani et al., "How clinical imaging can assess cancer biology," Insights into imaging, vol. 10, no. 1, 2019.

[7] S. Kumar, A. Mohan, and R. Guleria, "Biomarkers in cancer screening, research and detection: present and future: a review," Biomarkers, vol. 11, no. 5, pp. 385-405, 2006.

[8] E. Pickwell and V. Wallace, "Biomedical applications of terahertz technology," Journal of Physics D: Applied Physics, vol. 39, no. 17, pp. R301-R310, 2006.

[9] M. Danciu, T. Alexa-Stratulat, C. Stefanescu et al., "Terahertz spectroscopy and imaging: a cutting-edge method for diagnosing digestive cancers," Materials, vol. 12, no. 9, 2019.

[10] Y. Shen, T. Lo, P. F. Taday, B. E. Cole, W. R. Tribe, and M. C. Kemp, "Detection and identification of explosives using terahertz pulsed spectroscopic imaging," Applied physics letters, vol. 86, no. 24, article 241116, 2005.

[11] I. Duling and D. Zimdars, "Revealing hidden defects," Nature Photonics, vol. 3, no. 11, pp. 630-632, 2009.

[12] J. Federici and L. Moeller, "Review of terahertz and subterahertz wireless communications," Journal of Applied Physics, vol. 107, no. 11, article 111101, 2010.

[13] T. Kleine-Ostmann and T. Nagatsuma, "A review on terahertz communications research," Journal of Infrared, Millimeter, and Terahertz Waves, vol. 32, no. 2, pp. 143-171, 2011.

[14] C. Yu, S. Fan, Y. Sun, and E. Pickwell-MacPherson, "The potential of terahertz imaging for cancer diagnosis: a review of investigations to date," Quantitative imaging in medicine and surgery, vol. 2, no. 1, pp. 33-45, 2012.

[15] M.-A. Brun, F. Formanek, A. Yasuda, M. Sekine, N. Ando, and Y. Eishii, "Terahertz imaging applied to cancer diagnosis," Physics in Medicine \& Biology, vol. 55, no. 16, pp. 4615-4623, 2010.

[16] J. I. Goldstein, D. E. Newbury, J. R. Michael, N. W. Ritchie, J. H. J. Scott, and D. C. Joy, Scanning Electron Microscopy and X-Ray Microanalysis, Springer, 2017. 
[17] K. J. Ember, M. A. Hoeve, S. L. McAughtrie et al., "Raman spectroscopy and regenerative medicine: a review," NPJ Regenerative medicine, vol. 2, no. 1, pp. 1-10, 2017.

[18] S. Türker-Kaya and C. W. Huck, "A review of mid-infrared and near-infrared imaging: principles, concepts and applications in plant tissue analysis," Molecules, vol. 22, no. 1, 2017.

[19] D. Patterson, M. Schnell, and J. M. Doyle, "Enantiomer-specific detection of chiral molecules via microwave spectroscopy," Nature, vol. 497, no. 7450, pp. 475-477, 2013.

[20] J. El Haddad, B. Bousquet, L. Canioni, and P. Mounaix, "Review in terahertz spectral analysis," TrAC Trends in Analytical Chemistry, vol. 44, pp. 98-105, 2013.

[21] M. El-Shenawee, N. Vohra, T. Bowman, and K. Bailey, "Cancer detection in excised breast tumors using terahertz imaging and spectroscopy," Biomedical Spectroscopy and Imaging, vol. 8, no. 1-2, pp. 1-9, 2019.

[22] Y. Peng, C. Shi, Y. Zhu, M. Gu, and S. Zhuang, "Terahertz spectroscopy in biomedical field: a review on signal-to-noise ratio improvement," PhotoniX, vol. 1, no. 12, pp. 1-18, 2020.

[23] T. Li, H. Ma, Y. Peng et al., "Gaussian numerical analysis and terahertz spectroscopic measurement of homocysteine," Biomedical Optics Express, vol. 9, no. 11, pp. 5467-5476, 2018.

[24] J. P. Martin, C. S. Joseph, and R. H. Giles, "Continuous-wave circular polarization terahertz imaging," Journal of biomedical optics, vol. 21, no. 7, article 070502, 2016.

[25] T. Bowman, M. El-Shenawee, and L. K. Campbell, "Terahertz transmission vs reflection imaging and model-based characterization for excised breast carcinomas," Biomedical Optics Express, vol. 7, no. 9, pp. 3756-3783, 2016.

[26] S. Yamaguchi, Y. Fukushi, O. Kubota, T. Itsuji, T. Ouchi, and S. Yamamoto, "Origin and quantification of differences between normal and tumor tissues observed by terahertz spectroscopy," Physics in Medicine \& Biology, vol. 61, no. 18, pp. 6808-6820, 2016.

[27] F. Wahaia, I. Kasalynas, R. Venckevicius et al., "Terahertz absorption and reflection imaging of carcinoma-affected colon tissues embedded in paraffin," Journal of Molecular Structure, vol. 1107, pp. 214-219, 2016.

[28] M. R. Grootendorst, A. J. Fitzgerald, S. G. Brouwer de Koning et al., "Use of a handheld terahertz pulsed imaging device to differentiate benign and malignant breast tissue," Biomedical Optics Express, vol. 8, no. 6, pp. 2932-2945, 2017.

[29] T. Bowman, T. Chavez, K. Khan et al., "Pulsed terahertz imaging of breast cancer in freshly excised murine tumors," Journal of biomedical optics, vol. 23, no. 2, article 026004, 2018.

[30] Q. Cassar, A. al-Ibadi, L. Mavarani et al., "Pilot study of freshly excised breast tissue response in the $300-600 \mathrm{GHz}$ range," Biomedical Optics Express, vol. 9, no. 7, pp. 2930-2942, 2018.

[31] N. Vohra, T. Bowman, P. M. Diaz, N. Rajaram, K. Bailey, and M. El-Shenawee, "Pulsed terahertz reflection imaging of tumors in a spontaneous model of breast cancer," Biomedical physics \& engineering express, vol. 4, no. 6, article 065025, 2018.

[32] W.-G. Yeo, O. Gurel, C. L. Hitchcock, S. Park, K. Sertel, and N. K. Nahar, "Evaluation of cancer tissue morphology via $\mathrm{THz}$ spectroscopic imaging: human lung and small intestine malignancies," Infrared Physics \& Technology, vol. 97, pp. 411-416, 2019.

[33] K. Okada, K. Serita, Z. Zang et al., "Scanning laser terahertz near-field reflection imaging system," Applied Physics Express, vol. 12, no. 12, article 122005, 2019.
[34] T. Bowman, N. Vohra, K. Bailey, and M. el-Shenawee, "Terahertz tomographic imaging of freshly excised human breast tissues," Journal of Medical Imaging, vol. 6, no. 2, article 023501, 2019.

[35] Y. B. Ji, S. J. Oh, S. G. Kang et al., "Terahertz reflectometry imaging for low and high grade gliomas," Scientific Reports, vol. 6, no. 1, pp. 1-9, 2016.

[36] L. Wu, D. Xu, Y. Wang et al., "Study of in vivo brain glioma in a mouse model using continuous-wave terahertz reflection imaging," Biomedical Optics Express, vol. 10, no. 8, pp. 39533962, 2019.

[37] Y. Peng, X. Yuan, X. Zou et al., "Terahertz identification and quantification of neurotransmitter and neurotrophy mixture," Biomedical Optics Express, vol. 7, no. 11, pp. 4472-4479, 2016.

[38] L. Frattola, C. Ferrarese, N. Canal et al., "Characterization of the $\gamma$-aminobutyric acid receptor system in human brain gliomas," Cancer Research, vol. 45, no. 9, pp. 4495-4498, 1985.

[39] C. Cheng, Z. Zhu, S. Li et al., "Broadband terahertz recognizing conformational characteristics of a significant neurotransmitter $\gamma$-aminobutyric acid," RSC Advances, vol. 9, no. 35, pp. 20240-20247, 2019.

[40] N. D. Stefano, P. M. Matthews, and D. L. Arnold, "Reversible decreases in N-acetylaspartate after acute brain injury," Magnetic Resonance in Medicine, vol. 34, no. 5, pp. 721-727, 1995.

[41] W. Chen, Y. Peng, X. Jiang, J. Zhao, H. Zhao, and Y. Zhu, "Isomers identification of 2-hydroxyglutarate acid disodium salt (2HG) by terahertz time-domain spectroscopy," Scientific Reports, vol. 7, no. 1, pp. 12166-12166, 2017.

[42] E. Hattingen, P. Raab, K. Franz, F. E. Zanella, H. Lanfermann, and U. Pilatus, "Myo-inositol: a marker of reactive astrogliosis in glial tumors?," Journal Devoted to the Development and Application of Magnetic Resonance In vivo, vol. 21, no. 3, pp. 233-241, 2008.

[43] L. Yang, H. Sun, S. Weng et al., "Terahertz absorption spectra of some saccharides and their metal complexes," Spectrochimica Acta Part A: Molecular and Biomolecular Spectroscopy, vol. 69, no. 1, pp. 160-166, 2008.

[44] L. Wu and X. Qu, "Cancer biomarker detection: recent achievements and challenges," Chemical Society Reviews, vol. 44, no. 10, pp. 2963-2997, 2015.

[45] H. Ge, Y. Jiang, F. Lian, Y. Zhang, and S. Xia, "Quantitative determination of aflatoxin B1 concentration in acetonitrile by chemometric methods using terahertz spectroscopy," Food Chemistry, vol. 209, pp. 286-292, 2016.

[46] S. Lu, X. Zhang, Z. Zhang, Y. Yang, and Y. Xiang, "Quantitative measurements of binary amino acids mixtures in yellow foxtail millet by terahertz time domain spectroscopy," Food Chemistry, vol. 211, pp. 494-501, 2016.

[47] Y. Peng, C. Shi, M. Xu et al., "Qualitative and quantitative identification of components in mixture by terahertz spectroscopy," IEEE Transactions on Terahertz Science and Technology, vol. 8, no. 6, pp. 696-701, 2018.

[48] N. Qi, Z. Zhang, Y. Xiang, Y. Yang, and P. . B. Harrington, "Terahertz time-domain spectroscopy combined with fuzzy rule-building expert system and fuzzy optimal associative memory applied to diagnosis of cervical carcinoma," Medical Oncology, vol. 32, no. 1, 2015.

[49] H. Liu, Z. Zhang, X. Zhang et al., "Dimensionality reduction for identification of hepatic tumor samples based on terahertz time-domain spectroscopy," IEEE Transactions on Terahertz Science and Technology, vol. 8, no. 3, pp. 271-277, 2018. 
[50] W. Liu, R. Zhang, Y. Ling et al., "Automatic recognition of breast invasive ductal carcinoma based on terahertz spectroscopy with wavelet packet transform and machine learning," Biomedical Optics Express, vol. 11, no. 2, pp. 971-981, 2020.

[51] R. Zhang, L. Zhang, T. Wu et al., "Contrast-enhanced continuous-terahertz-wave imaging based on superparamagnetic iron oxide nanoparticles for biomedical applications," Optics Express, vol. 24, no. 8, pp. 7915-7921, 2016.

[52] T. Bowman, A. Walter, O. Shenderova, N. Nunn, G. McGuire, and M. El-Shenawee, "A phantom study of terahertz spectroscopy and imaging of micro- and nano-diamonds and nanoonions as contrast agents for breast cancer," Biomedical physics \& engineering express, vol. 3, no. 5, article 055001, 2017.

[53] Q. Huang, Y. Zou, S. Zhong et al., "Silica-coated gold nanorods with high photothermal efficiency and biocompatibility as a contrast agent for in vitro terahertz imaging," Journal of Biomedical Nanotechnology, vol. 15, no. 5, pp. 910-920, 2019.

[54] G. R. Musina, I. N. Dolganova, K. M. Malakhov et al., “Terahertz spectroscopy of immersion optical clearing agents: DMSO, PG, EG, PEG," in , Article ID 108000FMillimetre Wave and Terahertz Sensors and Technology XI, vol. 10800, Berlin, Germany, 2018.

[55] K. Yang, X. Yang, X. Zhao, M. Lamy de la Chapelle, and W. Fu, "THz spectroscopy for a rapid and label-free cell viability assay in a microfluidic chip based on an optical clearing agent," Analytical Chemistry, vol. 91, no. 1, pp. 785-791, 2019.

[56] E. M. Hassan, A. Mohamed, M. C. DeRosa et al., "High-sensitivity detection of metastatic breast cancer cells via terahertz chemical microscopy using aptamers," Sensors and Actuators B: Chemical, vol. 287, pp. 595-601, 2019.

[57] Z. Geng, X. Zhang, Z. Fan, X. Lv, and H. Chen, "A route to terahertz metamaterial biosensor integrated with microfluidics for liver cancer biomarker testing in early stage," Scientific Reports, vol. 7, no. 1, article 16762, pp. 1-11, 2017.

[58] K. Liu, R. Zhang, X. Chen, and E. Pickwell-MacPherson, "Detection of EGFR Protein Using Terahertz Metamaterial Biosensor," in 2018 43rd International Conference on Infrared, Millimeter, and Terahertz Waves (IRMMW-THz), pp. 1-2, Nagoya, Japan, 2018.

[59] C. Weisenstein, D. Schaar, A. Katharina Wigger, H. SchäferEberwein, A. K. Bosserhoff, and P. Haring Bolívar, "Ultrasensitive $\mathrm{THz}$ biosensor for PCR-free cDNA detection based on frequency selective surfaces," Biomedical Optics Express, vol. 11, no. 1, pp. 448-460, 2020. 